\title{
Deletion of the Bacillus anthracis capB homologue in Francisella tularensis subspecies tularensis generates an attenuated strain that protects mice against virulent tularaemia
}

\section{Correspondence \\ Rachel E. Dean \\ redean@dstl.gov.uk}

Received 15 February 2010

Accepted 19 July 2010

\author{
Stephen L. Michell,†‡ Rachel E. Dean,† Jim E. Eyles,§ \\ Margaret Gill Hartley, Emma Waters, Joann L. Prior, Richard W. Titballł \\ and Petra C. F. Oyston
}

Biomedical Sciences, Defence Science and Technology Laboratory, Porton Down, Salisbury,
Wiltshire SP4 OJO, UK

\begin{abstract}
As there is currently no licensed vaccine against Francisella tularensis, the causative agent of tularaemia, the bacterium is an agent of concern as a potential bioweapon. Although $F$. tularensis has a low infectious dose and high associated mortality, it possesses few classical virulence factors. An analysis of the $F$. tularensis subspecies tularensis genome sequence has revealed the presence of a region containing genes with low sequence homology to part of the capBCADE operon of Bacillus anthracis. We have generated an isogenic cap $B$ mutant of $F$. tularensis subspecies tularensis SchuS4 and shown it to be attenuated. Furthermore, using BALB/c mice, we have demonstrated that this $c a p B$ strain affords protection against significant homologous challenge with the wild-type strain. These data have important implications for the development of a defined and efficacious tularaemia vaccine.
\end{abstract}

\section{INTRODUCTION}

Due to its high infectivity and ability to cause severe disease and morbidity, Francisella tularensis, the causative agent of tularaemia, has been designated a category A biological agent (Saslaw et al., 1961b; Khan et al., 2000; Oyston et al., 2004). F. tularensis subspecies tularensis, previously known as type A (Olsufiev et al., 1959), is recognized as the most virulent biotype and has an infectious dose in humans of as low as 10 c.f.u. via the airborne route (McCrumb, 1961). F. tularensis subspecies holarctica, or type B, can also cause disease, but is less virulent than $F$. tularensis subspecies tularensis strains (Chen et al., 2003). At present, there is no licensed vaccine for the prevention of tularaemia although it has been demonstrated that an undefined live attenuated strain of $F$. tularensis subspecies holarctica confers reasonable protection against injected challenges with $F$.

†These authors contributed equally to this work.

$\ddagger$ Present address: School of Biosciences, Geoffrey Pope Building, University of Exeter, Stocker Road, Exeter EX4 4QD, UK.

§Present address: Vaccines, Global Research \& Development, Pfizer Limited, Sandwich, Kent CT13 9NJ, UK.

Abbreviations: IFN- $\gamma$, gamma interferon; IL-2, interleukin 2; MLD, median lethal dose; PGA, poly-D-glutamic acid; TNF- $\alpha$, tumour necrosis factor alpha.

A figure of serum sensitivity data is available as supplementary data with the online version of this paper. tularensis subspecies tularensis (Saslaw et al., 1961a, b). The fact that an attenuated mutant of $F$. tularensis can induce protective immunity suggests that the development of live vaccines based on defined mutations in virulence factors is a feasible approach to vaccine development. Few virulence determinants have been proposed and relatively little is known about virulence mechanisms of this bacterium (Larsson et al., 2005), although intracellular survival seems to be key to pathogenesis (Oyston, 2008). To date, the deletion of only seven loci, purMCD, $d s b A$ and $d s b B, w b t D E F$, FTT0918, FTT1103 and $c l p B$ has been shown to attenuate this highly virulent strain in a mouse model of infection (Pechous et al., 2008; Qin et al., 2008, 2009; Straskova et al., 2009; Thomas et al., 2007; Twine et al., 2005). F. tularensis subspecies tularensis lacks a type III secretion system, plasmids, toxins and other classical virulence factors thus prompting an empirical need to identify the virulence determinants of this pathogen.

An analysis of genome sequence data from attenuated and virulent strains of $F$. tularensis has revealed the presence of a conserved capBCA region with low levels of sequence homology to part of the capBCADE operon of Bacillus anthracis (Larsson et al., 2005; Makino et al., 1989; Ravel et al., 2009). In B. anthracis, the capBCADE operon encodes the synthetic machinery for the assembly of capsular polyD-glutamic acid (PGA), with deletion of this operon in $B$. anthracis resulting in an attenuated phenotype (Drysdale 
et al., 2005). While the deletion of cap genes in low virulence strains of $F$. tularensis has demonstrated a role in vivo (Su et al., 2007; Weiss et al., 2007), this has, however, not yet been investigated in the highly virulent $F$. tularensis subspecies tularensis. Our studies and those of others have shown that deletion of pilA and of $h f q$, in different species and in strains of $F$. tularensis, results in a more marked attenuation in the less virulent holarctica subspecies than in the more virulent tularensis subspecies (Meibom et al., 2009). Thus, we wished to study the phenotype of a CapBdeficient mutant of $F$. tularensis subspecies tularensis with respect to virulence. In a study by $\mathrm{Su}$ and colleagues, the cap locus containing $c a p B, c a p C$ and a putative transporter FTL_1412 (FTT0807) was all deleted (Su et al., 2007). As the function of FTL_1412 (FTT0807) has not yet been determined it cannot be ruled out that this mutation could have played a role in the observed attenuation of the mutant strain. In this study, we have demonstrated that a homologue of the B. anthracis cap biosynthetic locus plays a role in the virulence in the highly virulent SchuS4 strain. In addition, this $\operatorname{cap} B$ mutant was able to induce a protective immune response, suggesting that the cap locus may be a potential target for mutation in the development of a live attenuated tularaemia vaccine. As such, this mutation has already been combined into an avirulent mutant of $F$. tularensis subspecies tularensis strain SchuS4 (Conlan et al., 2010). This work conclusively demonstrates the role of this gene in the virulence of $F$. tularensis subspecies tularensis strain SchuS4.

\section{METHODS}

Bacterial strains, plasmids and media. The bacterial strains and plasmids used in this study are listed in Table 1. Strains of F. tularensis were cultured on blood cysteine glucose agar (BCGA) supplemented with $10 \mathrm{ml}(10 \% \mathrm{w} / \mathrm{v})$ histidine $\mathrm{l}^{-1}$, on modified Thayer Martin (TM) agar (BBL GC agar base) (Becton Dickinson) supplemented with $1 \%(\mathrm{w} / \mathrm{v})$ haemoglobin and $1 \%(\mathrm{v} / \mathrm{v})$ BBL IsoVitaleX (Becton Dickinson) and $50 \mu \mathrm{g}$ polymixin B sulphate $\mathrm{ml}^{-1}$, or in liquid culture in Chamberlain's defined media (Chamberlain, 1965) or modified cysteine partial hydrolysate (MCPH) broth. Escherichia coli were cultured on Luria-Bertani plates or broth. Chloramphenicol was added as required to growth media at $20 \mu \mathrm{g} \mathrm{ml}^{-1}$ for both E. coli and F. tularensis.

General DNA manipulation. DNA extraction and manipulation procedures were performed as described by Sambrook et al., 1989). Unless otherwise stated, enzymes for the manipulation of DNA and reagents for the detection of the DIG-labelled probe for Southern blotting were obtained from Roche Diagnostics. Chemicals were obtained from Sigma, and culture media were obtained from Oxoid. Oligonucleotides were supplied by MWG BioTech and are listed in Table 2.

\section{Construction of F. tularensis subspecies tularensis SchuS4} $\Delta \mathbf{c a p B}:$ : Cam. The suicide plasmid for inactivation of $c a p B$ was constructed as follows. Regions of DNA flanking the capB gene of $F$. tularensis were PCR amplified from $F$. tularensis subspecies tularensis SchuS4 genomic DNA using the primer pairs P1/P2 and P3/P4. The PCR products were digested with BstEII and ligated together. This ligation mixture was used as template in a PCR using the primer pair
$\mathrm{P} 1 / \mathrm{P} 4$. The $2085 \mathrm{bp}$ product from this reaction was cloned into the plasmid pGEM-T Easy to give the plasmid pSMP32. The chloramphenicol resistance cassette (Cam) was PCR amplified from the plasmid pKK202 (Norqvist et al., 1996) with the primer pair CamF/ CamR and cloned into the BstEII restriction of pSMP32 to generate pSMP39. This mutated allele of $c a p B$ was excised from pSMP39 by $M l u \mathrm{I}$ digestion and cloned into the MluI site of pSMP22 to give pSMP42. The suicide plasmid pSMP42 for deletion of $c a p B$ was electroporated into the E. coli mobilizing strain S17ipir (Simon et al., 1983). The plasmid was then introduced from the mobilizing strain to F. tularensis subspecies tularensis SchuS4 by conjugal transfer, as described by Golovliov et al. (2003). Transconjugants were selected on chloramphenicol and merodiploids arising from chromosomal integration of the suicide plasmid were resolved by plating on TM agar containing $5 \%(\mathrm{w} / \mathrm{v})$ sucrose. Colonies were confirmed as mutants by Southern blotting. Briefly, genomic DNA of wild-type and SchuS4 $4 c a p B$ ::Cam mutants was digested with $M l u \mathrm{I}$ and $\mathrm{NcoI}$, separated by agarose gel electrophoresis and transferred to a nylon membrane. The MluI insert of pSMP32 was labelled with DIG-11dUTP during PCR amplification with the primer pair P1/P4 and used as a probe to hybridize to the membrane. DNA fragments to which the probe hybridized were detected by chemiluminescence [CSPD - disodium 3-(4-methoxyspiro[1,2-dioxetane-3,2'-(5'-chloro)tricyclo(3.3.1.13,7)decan]-4-yl)phenylphosphate - substrate, $30 \mathrm{~min}$ exposure, X-ray film].

The use of chloramphenicol as a marker was approved for this specific study. All work undertaken in the UK is reviewed locally by a genetic manipulation safety committee on a project-by-project basis and is also approved nationally by our health and safety authorities.

Real-time PCR. Bacteria were cultured in MCPH broth at $37{ }^{\circ} \mathrm{C}$ with shaking at 180 r.p.m. until cells were growing exponentially. RNA extraction was performed using an RNeasy midi preparation kit (Qiagen). Contaminating DNA was removed using Turbo DNA-free (Ambion) and mRNA was reverse transcribed using an Omniscript reverse transcription kit (Qiagen). Oligonucleotide primers and probes were designed and supplied by Applied Biosystems. The probe used for the analysis of gene expression was located within the target gene. These sequences are shown in Table 2. Each reaction contained $1.25 \mu \mathrm{l}$ TaqMan gene expression assay, $12.5 \mu \mathrm{l}$ TaqMan PCR master mix, $6.25 \mu \mathrm{l}$ nuclease-free water (Applied Biosystems) and $5 \mu \mathrm{l} \mathrm{cDNA}(500 \mathrm{ng}$ ). TaqMan analysis was performed in a MicroAmp optical 96-well plate using the ABI Prism 7000 sequence detection system (Applied Biosystems). Assays were performed in triplicate on cDNA samples, using dnaA as an endogenous control (Livak \& Schmittgen, 2001) with a standard cycle protocol.

Intramacrophage survival assay. J774A murine macrophages were infected at an m.o.i. of 10 and incubated at $37^{\circ} \mathrm{C}$ for $30 \mathrm{~min}$. Extracellular bacteria were killed with $10 \mu \mathrm{g}$ gentamicin $\mathrm{ml}^{-1}$ for $30 \mathrm{~min}$. Cultures were maintained in L-15 tissue culture medium (Sigma-Aldrich) containing $2 \mu \mathrm{g}$ gentamicin $\mathrm{ml}^{-1}$ at $37^{\circ} \mathrm{C}$, until harvesting at various times post-infection. Macrophages were

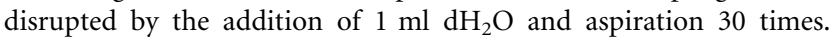
Bacteria were enumerated by plating them on to BCGA and incubating for 3 days at $37^{\circ} \mathrm{C}$.

In vivo studies. Female BALB/c mice (6-8 weeks old; Charles River Laboratories) were injected subcutaneously with $100 \mu \mathrm{l}$ PBS containing $10^{4}$ c.f.u. F. tularensis subspecies tularensis SchuS4, F. tularensis subspecies tularensis SchuS $4 \Delta$ capB::Cam or F. tularensis subspecies holarctica LVS. Several investigators have determined that the median lethal dose (MLD) of F. tularensis subspecies tularensis is $<10$ c.f.u. in the $\mathrm{BALB} / \mathrm{c}$ mouse, irrespective of route of administration (Saslaw et al., 1961a, b). For protection studies, immunized and naive mice were challenged on day 56 post-vaccination with $10^{3}$ c.f.u. F. tularensis 
Table 1. Bacterial strains and plasmids used in this study

\begin{tabular}{|c|c|c|}
\hline Bacteria or plasmid & Characteristic & Reference or source \\
\hline \multicolumn{3}{|l|}{ Strains } \\
\hline E. coli S17 $\lambda$ pir & $\begin{array}{l}\text { thi thr leu tonA lacY supE recA::RP4-2-Tc:: Mu, } \\
\text { Kn::Tn7 }\end{array}$ & Simon et al. (1983) \\
\hline F. tularensis subsp. tularensis SchuS4 & Virulent strain & Human ulcer, 1941, Ohio, FSC237 \\
\hline F. tularensis subsp. holarctica LVS & Avirulent strain - basis of attenuation not known & $\begin{array}{l}\text { Original NDBR lot } 4 \text { vaccine ampoule } \\
\text { produced during the } 1960 \mathrm{~s}\end{array}$ \\
\hline \multicolumn{3}{|l|}{ Plasmids } \\
\hline pGEM-T Easy & Commercial vector & Promega \\
\hline pSMP32 & pGEM-T Easy containing $c a p B$ flanking regions & This study \\
\hline pSMP22 & Mobilizable suicide vector & Thomas et al. (2007) \\
\hline pSMP42 & $\begin{array}{l}\text { Plasmid for deletion of } c a p B \text {, contains insert from } \\
\text { pSMP39 cloned into pSMP22 }\end{array}$ & This study \\
\hline
\end{tabular}

subspecies tularensis SchuS4 via the subcutaneous route. For colonization studies, groups of four mice were killed at 3, 7, 14 and 31 days following inoculation with the bacteria. Spleens were removed and the number of bacteria per spleen determined by serial dilution in PBS followed by microbiological culture on BCGA agar plates for $96 \mathrm{~h}$ at $37^{\circ} \mathrm{C}$. All procedures were carried out in accordance with UK Home Office guidelines. Animals were closely observed over a 35 day period for the development of clinical signs. Humane end points were strictly observed, so that if an animal displayed irreversible signs of tularaemia, it was promptly culled, thus avoiding undue distress.

Inactivation of bacteria. Overnight cultures of $F$. tularensis subspecies holarctica LVS grown on BCGA at $37^{\circ} \mathrm{C}$ were inactivated by irradiation with $30 \mathrm{kGy}$ per vial (Isotron). Before and after irradiation, $10 \%$ of the vial contents were removed and plated on BCGA to assess viability. The concentration of protein in each vial was ascertained using a bicinchoninic acid protein assay (Pierce). Irradiated bacteria were stored at $-20{ }^{\circ} \mathrm{C}$ prior to use in immunization studies. F. tularensis subspecies tularensis SchuS4 was inactivated by heating at $65{ }^{\circ} \mathrm{C}$ for $1 \mathrm{~h}$.

\begin{abstract}
Humoral and cellular responses following immunization with the SchuS4 $\Delta$ capB : : Cam mutant. Bacteria were grown for $48 \mathrm{~h}$ at $37{ }^{\circ} \mathrm{C}$ on modified TM agar. Female BALB/c mice (6-8 weeks old) were injected subcutaneously with $100 \mu \mathrm{PBS}$ containing $10^{4}$ c.f.u. $F$. tularensis subspecies tularensis SchuS $4 \Delta c a p B::$ Cam, F. tularensis subspecies holarctica LVS or PBS alone. Blood was sampled from immunized and naive mice from the tail vein on day 34 . Serum was analysed for anti-F. tularensis subspecies holarctica LVS antibodies using standard ELISA methodology. Briefly, individual serum samples were dispensed into microtitre plates pre-coated with either irradiated LVS or heat-killed $F$. tularensis subspecies tularensis SchuS4 $(5 \mu \mathrm{g}$ bacterial protein $\mathrm{ml}^{-1}$ in PBS). Binding of serum antibody was detected with peroxidase-labelled secondary antibody to mouse IgG1 and IgG2a (Harlan-SeraLab). To facilitate a comparison of one subclass titre with another, standard solutions of each subclass antibody (Harlan-SeraLab) in the range of $0.2-50.0 \mathrm{ng} \mathrm{ml}^{-1}$ were
\end{abstract}

Table 2. Oligonucleotides used in this study

Restriction sites are indicated by underlined sequences.

\begin{tabular}{|c|c|}
\hline Primer & Sequence \\
\hline $\mathrm{P} 1$ & 5'-CTGACGCGT-AGGCAGTGTGGTTATGGGTAG-3' \\
\hline $\mathrm{P} 2$ & 5'-GACGGTAACC-CAAATACGACGACAATTAAC-3' \\
\hline P3 & 5'-CTGGGTAACC-TCCAGCAAACTCTTATATTC-3' \\
\hline $\mathrm{P} 4$ & 5'-TAGACGCGT-ACCCAATCAACCCAGTACAAG-3' \\
\hline $\operatorname{CamF}$ & 5'-GCTGGTTACC-TAAGAGGTTCCAACTTTCAC-3' \\
\hline $\operatorname{Cam} \mathrm{R}$ & 5'-CTAGGTTACC-TTTAAGGGCACCAATAACTG-3' \\
\hline capC F ABI & 5'-GGACTATCTACAGGTGGTATGGTTGT-3' \\
\hline capC R ABI & 5'-CGTCTACCGTAAATAATCATAAATTTAGACATAAATCTGA-3' \\
\hline capC probe & 5'-CAGGAGCCCCCATCTC-3' \\
\hline$d n a A \mathrm{~F} \mathrm{ABI}$ & 5'-CTCAAAAAAGCTCATGATTTAGGTCAGAA-3' \\
\hline dnaA R ABI & 5'-AGCACCTTCTAGTTCTCTGACATTAGT-3' \\
\hline dnaA probe & 5'-CAGCAGCTTTTATTGC-3' \\
\hline
\end{tabular}


assayed. The standard curves generated enabled determination of the mean concentration of each IgG subclass in serum derived from the various treatment groups. At 40 days following immunization, groups of four immunized or naive mice were sacrificed and their spleens removed. Single cell suspensions of spleen cells were prepared in culture media (RPMI 1640) (Sigma) supplemented with $10 \%$ heat inactivated fetal bovine serum (Sigma), $1 \%$ penicillin/streptomycin/ glutamine (Sigma) and $50 \mu \mathrm{M} 2$-mercaptoethanol (Sigma). Cells were stimulated overnight in triplicate with either heat-killed $F$. tularensis subspecies tularensis SchuS4 $\left(5 \mu \mathrm{g}\right.$ protein $\left.\mathrm{ml}^{-1}\right)$ in supplemented RPMI 1640 or supplemented RPMI 1640 alone. Interleukin 2 (IL-2), tumour necrosis factor alpha (TNF- $\alpha$ ) and gamma interferon (IFN- $\gamma$ ) secretion from the cells was determined using cytokine bead array technology (BD Biosciences).

Statistics. Statistical differences in antibody response were determined using ANOVA, Kruskal-Wallis and Student-Newman-Keuls tests. Survival data were analysed using Kaplan-Meier plots and a Mantel-Haenszel log-rank test.

\section{RESULTS}

\section{F. tularensis subspecies tularensis contains homologues of the $B$. anthracis capB and capC genes}

During annotation of the genome of $F$. tularensis subspecies tularensis SchuS4 (Larsson et al., 2005), we identified two adjacent genes, FTT0805 and FTT0806, that displayed 36 and $33 \%$ sequence identity at the amino acid level, with the CapB and CapC proteins, respectively, of $B$. anthracis and Staphylococcus epidermidis (Fig. 1). In B. anthracis, CapB and CapC have been shown to be responsible for the synthesis of PGA, while CapA and CapE are involved in transportation (Candela \& Fouet, 2006). The synteny between the F. tularensis cap locus, and the cap locus of $B$. anthracis and S. epidermidis, is not completely preserved with the notable absence in $F$. tularensis of homologues to capADE, the genes required for the secretion and anchoring of PGA (Fig. 1a). It has been proposed that the gene downstream of capC (FTT0807) be renamed capF (or pgsF if PGA is not anchored to the cell wall) as it may play a role in transport similar to the roles of capA and capE (Candela et al., 2009). In a report of the production of PGA by the Gram-negative bacterium Fusobacterium nucleatum, PGA appeared to be secreted to the culture medium (Candela et al., 2009) suggesting that the gene in Fusobacterium nucleatum does not encode a functional homologue of CapD of $B$. anthracis; the latter being involved in anchoring PGA to the cell wall (Candela \& Fouet, 2005). Indeed, the gene identified in F. tularensis as a homologue of the capD of $B$. anthracis (Su et al., 2007), which has less sequence homology than the Fusobacterium nucleatum homologue, has recently been shown to be ggt encoding a gamma glutamyltransferase involved in the acquisition of cysteine from glutathione in the LVS strain (Alkhuder et al., 2009), and more recently we have demonstrated this in the SchuS4 strain (H. LeButt, P. M. Ireland, R. M. Thomas \& P. C. F. Oyston, personal communication), supporting its original annotation as ggt rather than capD (Larsson et al., 2005).

\section{Construction of a capB-deficient strain of $F$. tularensis subspecies tularensis SchuS4}

To investigate the role of cap genes in F. tularensis subspecies tularensis, the capB gene (FTT0805) was chosen as a target for inactivation on the basis of its sequence homology to the CapB protein encoded by the cap virulence locus of $B$. anthracis strain 'Ames' and S. epidermidis 1457 (Kocianova et al., 2005; Makino et al., 1989) (Fig. 1b). Insertion of a Cam cassette resulted in a 1073 bp deletion of the cap $B$ gene, with 6 and 35 amino acids remaining at the $\mathrm{N}$ - and $\mathrm{C}$-terminus, respectively. Allelic replacement mutants were confirmed by Southern blotting as demonstrated by the two predicted hybridizing fragments of 12.5 and $3.2 \mathrm{~kb}$ contained in the SchuS4 $\Delta c a p B$ :: Cam mutant (Fig. 2). Our local advisory committees on dangerous pathogens and genetic modification prohibit the use of two antibiotic resistance markers in F. tularensis subspecies tularensis SchuS4; thus, preventing us from generating a complemented mutant strain. Therefore, to investigate whether insertion of the Cam cassette resulted in a disruption of downstream gene expression, expression of $c a p C$, the gene immediately downstream of $c a p B$, was examined by reverse transcription RTPCR. RNA was isolated from exponentially growing cultures of $F$. tularensis subspecies tularensis SchuS4 strain and SchuS4 $\triangle$ cap $B::$ Cam, and reverse transcribed. Expression of cap $C$ was confirmed using the method described by Livak \& Schmittgen (2001).

\section{F. tularensis subspecies tularensis SchuS4 $\triangle$ capB : : Cam mutant is attenuated and induces a protective immune response}

Inactivation of $c a p B$ did not impair the growth rate of $F$. tularensis subspecies tularensis SchuS4 $\triangle$ capB::Cam in $\mathrm{MCPH}$ broth, and replication within J774 murine macrophage cells was not significantly affected (Fig. 3). In addition, we did not observe a difference in serum sensitivity in SchuS4 $\triangle c a p B::$ Cam, compared to wildtype (Supplementary Fig. S1 available with the online journal). In order to determine whether $c a p B$ is required for virulence in a murine model of infection, mice were infected subcutaneously with $F$. tularensis subspecies tularensis SchuS4 or SchuS4 $\triangle c a p B$ :: Cam. Mice infected with 120 c.f.u. of strain SchuS4 succumbed to infection by day 4 post-infection. In contrast, $100 \%$ of mice infected with $1.6 \times 10^{4}$ c.f.u. of SchuS4 $\triangle \mathrm{cap} B$ : : Cam survived, and $80 \%$ of mice infected with $1.6 \times 10^{5}$ or $1.6 \times 10^{6}$ c.f.u. of SchuS $4 \Delta c a p B$ ::Cam survived (Fig. 4a). Therefore, the MLD was assumed to be $>1.6 \times 10^{6}$. This significant $(P=0.0026)$ level of survival following deletion of a single gene demonstrates that $c a p B$ is important for the virulence of $F$. tularensis subspecies tularensis. Our data indicate that the level of protection afforded by the SchuS $4 \Delta c a p B:$ :Cam mutant is comparable to that of LVS as mice immunized 
(a)

Bacillus anthracis AMES ancestor

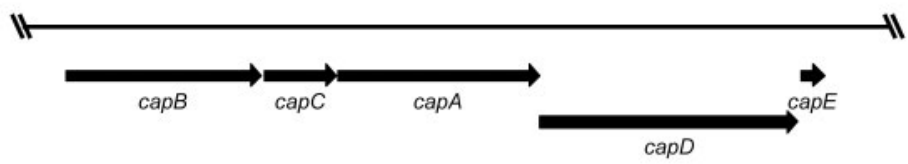

Francisella tularensis subspecies tularensis SchuS4

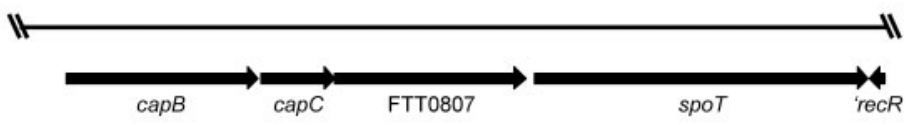

Staphylococcus epidermidis 1457

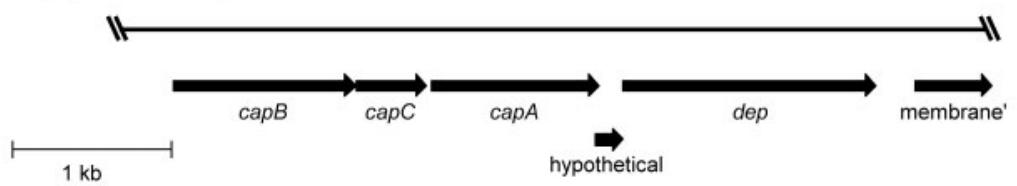

(b) BACaPB 1 ----MIFIIGICTVFLIIYGIWEQRCHQKRLNSIPIRVNINGIRGKSTVTRLITGVVQEA SECaPB 1 ----MLLIIA-CVALILWLGIKEKKRHANRLEKIPLRININGIRGKSTITRMAYSVLRED \# $\#$ \# \# \#\# \# \#\#T\#\#TT

BACaPB 57 KYKTVGKTTGTSARMIYWFTDEEQPIKRRKEGPNIGEQRRVVKEAADLEAEALICECMAV FTCaPB 61 GYRTVAKTTGTLARYID-VDGSETPVFRIGFS-NIAEQVKIMFKARRAKADAIVIECMAL SECaPB 56 QYRVVGKTTGTDARMLYWFTEKEYPVIRKPQGANIGEQRDIIRKVVKQKANALVNECMAV

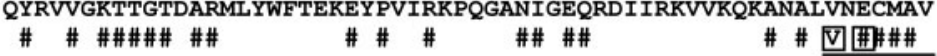

BACAPB 117 QPDYQIIFQNKMIQANVGVIVNVLEDHMDVMGPTLDEVAEAFTATIPYNGHLVTIESEYL FTCaPB 119 QPLLQSLCELKLIKATHGVLTNARPDHLDVMGPTERDVAKALAATVPVGAKYFTAEDIHL SEcapB 116 NPDYQITFQNDLVKANIGVIVNVMEDHMDVLGPTLKDVAQAFTATIPYNGKLVVMKDNYT

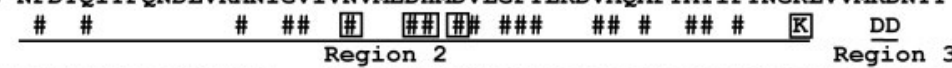

BACAPB 177 DYFKEVAEERNTKVIVA---DNSRISEEFLRKFDYMVFPDNASLALAVAEALGIDEETAF FTCaPB 179 DFFEYACKDRGSELIAATAQDAEKISDEEINKFVYSEFKINVALALKVIDDLGIPREIAL SEcapB 176 SFFAKEAKKRNSELIVV---DKDVIPESYLRKFDYLVFPDNVAIVLGIAQAVGVDEETAL

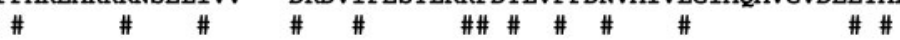

BACAPB 234 RGMLNAHPDPGAMRITRFADQSKPAFFVNGFAANDPSSTLRIWERVDDFGYSNL-APIVI FTCaPB 239 KGMWEATPDPGAMTEYNFNIKNAEINFANAFAANDPVSTKMLWDKLC-AKYSGCDKKVLV SECaPB 233 QGMLNAPADPGAVRIKYFHANRTKNVFVNAFAANEPQSTKAILNKVESYNYPYD-KKIII

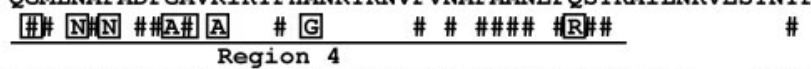

BACAPB 293 MNCRPDRVDRTEQFARDVLPYIKAEIVIAIGETTAPITSAFEKG----DIPTQEYWNLEG FTCaPB 298 VNCRDDREDRSKQMAEAALGWQKQDLIVLIGTGTEVFTSFYKKYAKSLNKPMTKVIVCEE SECaPB 292 LNCRSDRVDRTQLFVDNFLGEVDYDVLICTGKSTQMVTQFMET-------MPEKTY INYEG

$$
\text { \#\#\# \#\# \#\# }
$$

$\# \# \# \#$

BACAPB 349 WSTSEIMSRM---RPYLKNRIVYGVGNIHGAAEPLIDMIMEEQIGKKOAKVI FTCAPB 358 MTPIQILEKTVDSNP-ANSYILVGVGNIKDIGMELVDYC--DTSHKKKHNLSECapB 346 RDFVEIEKGI---LHEAENALVFCVGNIHGPGGRIAEFI--EGIE-------

Fig. 1. (a) Schematic representation of the cap locus from B. anthracis 'Ames Ancestor', F. tularensis subspecies tularensis SchuS4 and S. epidermidis 1457. (b) Global alignment of the amino acid sequences of CapB from B. anthracis, F. tularensis and $S$. epidermidis performed by Clone manager using a BLOSUM 62 scoring matrix.

with $10^{4}$ c.f.u. of SchuS $4 \Delta c a p B::$ Cam were solidly protected when challenged subcutaneously with $10^{3}$ c.f.u. wild-type $F$. tularensis subspecies tularensis SchuS4 56 days later $(P=0.0001)$ (Fig. 4b).

When the kinetics of bacterial clearance were examined, none of the mice infected with strain SchuS4 survived longer than 5 days post-inoculation, which precluded determination of splenic bacterial burdens on days 7, 14 and 31 post-inoculation (Fig. 5). However, at day 3 postinoculation there was a highly significant $(P<0.001)$ difference in the numbers of bacteria in the spleens of mice infected with strains SchuS4 and SchuS4 $\Delta c a p B:$ :Cam; as mice infected with the mutant had 


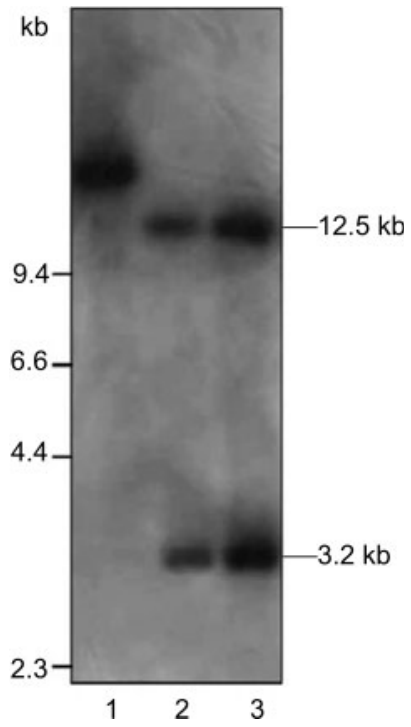

Fig. 2. Southern blot of genomic DNA from wild-type and SchuS $4 \Delta$ capB : : Cam mutant strains of $F$. tularensis subspecies tularensis. Genomic DNA strain SchuS4 (lane 1) and of two SchuS4 $\Delta$ cap $B$ : : Cam mutants (lanes 2 and 3 ) were digested with Mlul and $\mathrm{Ncol}$, separated by agarose gel electrophoresis and transferred to a nylon membrane. DNA fragments to which the probe hybridized were detected by chemiluminescence.

substantially lower numbers of bacteria in their spleens. No viable bacteria were detected in the spleens of animals infected with SchuS $4 \Delta c a p B$ ::Cam at 31 days post-inoculation; thus, indicating that the animals had effectively cleared the mutant.

\section{Characterizing the protective immune response induced by $F$. tularensis subspecies tularensis SchuS4 $\triangle$ capB : : Cam}

In our studies, mice immunized with the SchuS4 $\Delta$ capB : : Cam mutant responded with specific antibody and cell-mediated responses, similar to that observed for LVS, as assessed by immunoassay. ELISA confirmed that mice dosed with LVS or SchuS4 $\Delta c a p B$ ::Cam responded with robust serum IgG2a antibody responses (Fig. 6). Significantly increased quantities of IL-2 and IFN- $\gamma$ were secreted when spleen cells from animals immunized with SchuS4 $\Delta c a p B$ : Cam or LVS were restimulated in vitro with inactivated $F$. tularensis subspecies tularensis SchuS4 $(P<0.05)$ (Fig. 7a, b). No significant IL-2 or IFN- $\gamma$ secretion was detected when spleen cells from animals immunized with SchuS4 $\Delta c a p B$ ::Cam or LVS were cocultured with media alone, thereby confirming a Francisellaspecific cytokine recall response. TNF- $\alpha$ secretion during ex vivo stimulation with inactivated $F$. tularensis subspecies tularensis SchuS4 was also statistically increased $(P<0.05)$ when cells were derived from mice immunized with SchuS4 4 capB : : Cam or LVS (Fig. 7c). However, TNF- $\alpha$ was also secreted by spleen cells derived from PBS-treated mice
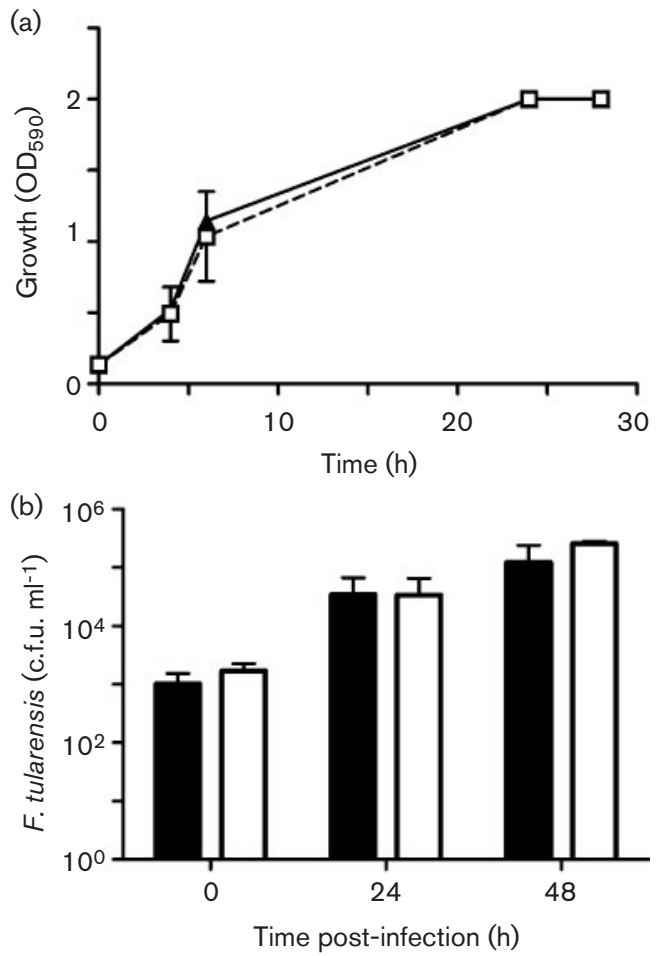

Fig. 3. (a) Growth of SchuS4 ( $(\boldsymbol{\Delta})$ and SchuS4 $\Delta$ cap $B$ : : Cam ( $\square$ ) in $\mathrm{MCPH}$ broth. Cultures were incubated at $37{ }^{\circ} \mathrm{C}$ with shaking at 180 r.p.m. and the optical density (at $590 \mathrm{~nm}$ ) measured at various time points. Data are presented as means and SEM of triplicate cultures. (b) Replication of SchuS4 (black bars) and SchuS4 $\Delta$ capB::Cam (white bars) in $\mathbf{J 7 7 4}$ murine macrophage cells. Cells were infected at a multiplicity of 10 , and intracellular bacteria harvested and enumerated at 0, 24 and $48 \mathrm{~h}$ post-infection. Data are presented as the mean and SEM of triplicate experiments.

during ex vivo stimulation. This probably reflects TNF- $\alpha$ production by macrophages and dendritic cells following activation by Toll-like receptor agonists present in the heatinactivated F. tularensis subspecies tularensis SchuS4.

\section{DISCUSSION}

In the last 40 years, there have been few reports of capsule production by F. tularensis (Cherwonogrodzky et al., 1994; Hood, 1977; Sandström et al., 1988). Furthermore, the reports of capsule production by Francisella are contrasting, making it difficult to ascertain its nature. One of the first, and most detailed, reports on capsule of the $F$. tularensis stated that it was observed as an electrontransparent layer, and that analysis of this material revealed a carbohydrate content of $21 \%$ and a fatty acid content of $51 \%$ (Hood, 1977). In contrast, subsequent studies described a Francisella capsule as an electron-dense layer; although no compositional studies were conducted on this material (Sandström et al., 1988). During the course of this study, we attempted several methods to visualize capsules 
(a)

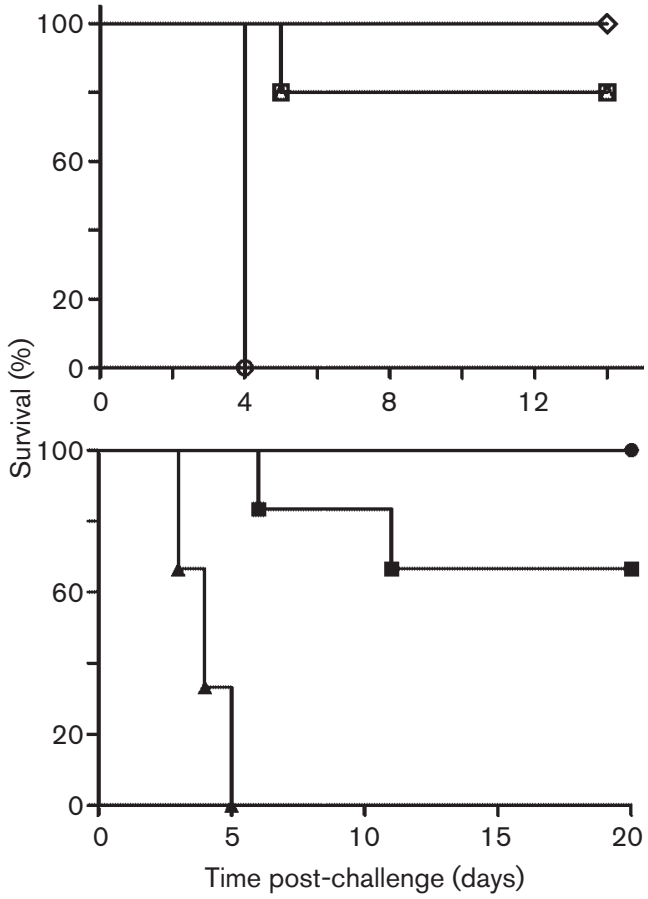

Fig. 4. (a) Survival following infection of BALB/c mice with 120 c.f.u. F. tularensis subspecies tularensis SchuS4 $(\bigcirc), 1.6 \times 10^{4}$ c.f.u. F. tularensis subspecies tularensis SchuS4 $\Delta$ capB::Cam $(\diamond), \quad 1.6 \times 10^{5}$ c.f.u. $F$. tularensis subspecies tularensis SchuS $4 \Delta$ cap $B$ : : Cam $(\triangle)$ or $1.6 \times 10^{6}$ c.f.u. F. tularensis subspecies tularensis SchuS4 $\Delta$ capB::Cam $(\square)$. Groups of five mice were infected by the subcutaneous route and monitored for 14 days. (b) Survival following infection of BALB/c mice with $10^{3}$ c.f.u. F. tularensis subspecies tularensis SchuS4 by the subcutaneous route. At 56 days prior to the challenge, mice were injected subcutaneously with either $10^{4}$ c.f.u. $F$. tularensis subspecies tularensis SchuS4 $\Delta$ capB::Cam $(\boldsymbol{\square})(n=5), 10^{4}$ c.f.u. F. tularensis subspecies holarctica LVS $(\mathbf{\Lambda})(n=5)$ or PBS $(\bullet)(n=6)$.

on F. tularensis grown under a range of conditions, but no such structure was observed (data not shown). Therefore, it is still not possible to say that the cap operon of $F$. tularensis is involved in capsule production, as is the case for the cap operon of $B$. anthracis, which is involved in the biosynthesis of a PGA capsule. As mentioned, it is possible that $F$. tularensis produces PGA through the function of the products of the $c a p B$ and $c a p C$ genes, although it might not be presented as a capsular structure. Although it has been proposed that FTT0807 may function as the cognate transporter in place of a CapA homologue (Candela et al., 2009; Su et al., 2007), preliminary analysis of SchuS4 and SchuS4 $\triangle c a p B$ ::Cam extracts by NMR failed to identify PGA in culture supernatant precipitates and cell extracts (data not shown); therefore, it remains to be definitively confirmed that the cap locus is involved in PGA production. As a result, we focussed on evaluating the effect of mutation of the $c a p B$ gene on virulence.

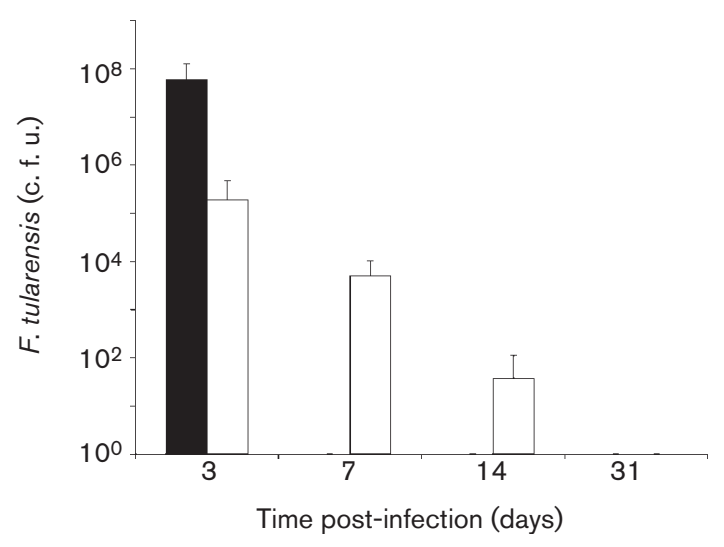

Fig. 5. Clearance kinetics following infection of $B A L B / c$ mice with $10^{4}$ c.f.u. strain SchuS4 (black bar) or $10^{4}$ c.f.u. SchuS4 $\Delta$ capB::Cam (white bars) by the subcutaneous route. Data are means $( \pm S D)$ from four individual spleens per treatment at each time point. No mice infected with $10^{4}$ c.f.u. F. tularensis subspecies tularensis SchuS4 survived beyond day 5 .

The detection of homologues of the cap locus of $B$. anthracis in $F$. tularensis subspecies tularensis suggested that they might also play a role in the virulence in this organism. Studies in low virulence strains of $F$. tularensis have demonstrated the cap locus is essential for virulence. In this study, the finding that a capB mutant of $F$. tularensis subspecies tularensis exhibits an MLD $>1.6 \times 10^{6}$ relative to $<10$ for the parent strain confirms that this previously Gram-positive bacteria specific virulence factor is also a virulence factor in the highly virulent Gram-negative $F$. tularensis subspecies tularensis.

The generation of defined attenuated mutants of $F$. tularensis subspecies tularensis is a rational approach in

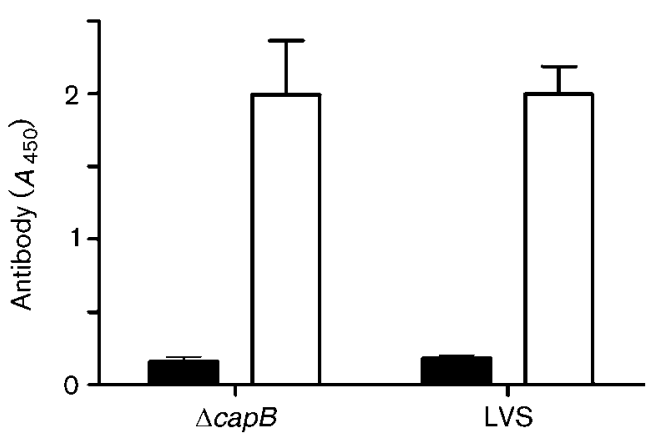

Fig. 6. Levels of specific anti-F. tularensis subspecies tularensis SchuS4 lgG1 (black bars) and lgG2a (white bars) antibodies in the serum of BALB/c mice infected subcutaneously 34 days previously with $10^{4}$ c.f.u. SchuS $4 \Delta$ cap $B$ : : Cam or $10^{4}$ c.f.u. LVS. Data are means $( \pm S E M)$ from five individual mice. Analysis of serum from PBS-treated mice gave mean absorbance (at $450 \mathrm{~nm}$ ) measurements of 0.075 and 0.070 for $\lg G 1$ and $\lg G 2 a$, respectively. 

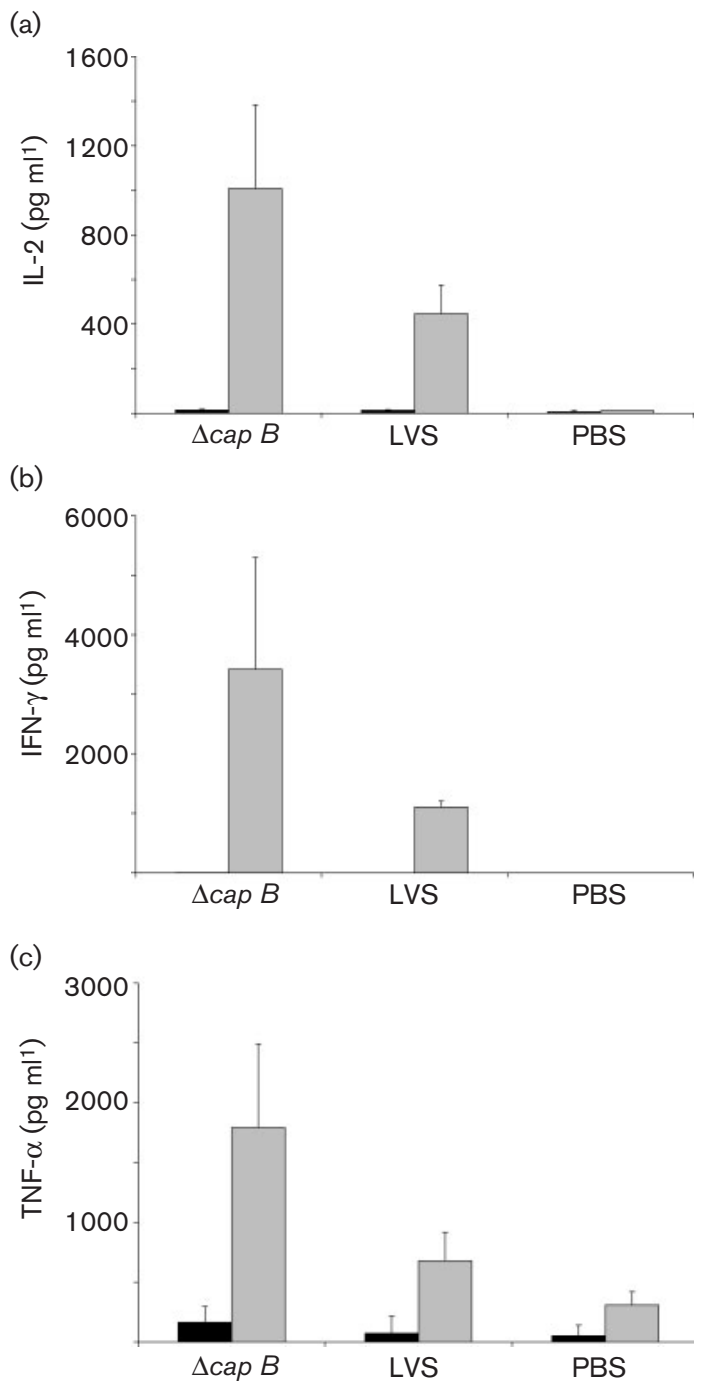

Fig. 7. (a) IL-2 secretion from spleen cells co-cultured in vitro with heat-killed $F$. tularensis subspecies tularensis SchuS4 (grey bars) or media alone (black bars). (b) IFN- $\gamma$ secretion from spleen cells co-cultured in vitro with heat-killed $F$. tularensis subspecies tularensis (grey bars) or media alone (black bars). (c) TNF- $\alpha$ secretion from spleen cells co-cultured in vitro with heat-killed $F$. tularensis subspecies tularensis SchuS4 (grey bars) or media alone (black bars). Spleen cells were isolated from BALB/c mice that had been injected subcutaneously with PBS or infected 40 days earlier with $10^{4}$ c.f.u. SchuS $4 \Delta$ cap $B$ : : Cam or $10^{4}$ c.f.u. LVS by the subcutaneous route. Data are means $( \pm S D)$ from four individual mice per treatment group.

tularaemia vaccine development (reviewed by Oyston, 2009); moreover, it is interesting to note that strains of $B$. anthracis that are deficient in the cap locus have been used in humans as vaccines against anthrax (Merabishvili et al., 2006). In our studies, mice infected with SchuS4 $\triangle$ capB::Cam and subsequently challenged with the fully virulent strain SchuS4 were protected. The mutant did not result in as high levels of colonization as the wild-type, but persisted at detectable levels for at least 2 weeks. Complete clearance was observed by a month postimmunization. Mice infected with the SchuS $4 \Delta c a p B:$ :Cam mutant responded with specific antibody and cell-mediated responses as adjudged by immunoassay. As was the case with LVS, SchuS4 $\triangle c a p B$ :: Cam immunized mice developed an IgG2a-dominated $F$. tularensis specific antibody response. Previous work with inactivated LVS has indicated a link between protection and the development of IgG2a antibodies with specificities for certain proteins within the $F$. tularensis proteome (Eyles et al., 2007). Similarly, mice immunized with LVS or SchuS4 $\triangle$ cap B: : Cam had significant numbers of F. tularensis specific IFN- $\gamma$ and IL-2 secreting T cells in their spleens, as compared with non-immunized animals. This is potentially important as, in common with many other intracellular pathogens, cell-mediated responses are known to play an important role in immunity to virulent forms of tularaemia, as demonstrated by passive transfer and cell depletion studies in rats and mice (Anthony et al., 1989; Conlan et al., 1994; Fortier et al., 1991; Tärnvik, 1989). Similarly, it is known that individuals recovering from tularaemia often demonstrate pronounced delayed-type hypersensitivity reactions (Burke, 1977).

We have demonstrated that $F$. tularensis subspecies tularensis possesses $c a p B$, a homologue of the B. anthracis virulence gene, a finding that, to the best of our knowledge, has not previously been reported in any other pathogenic Gram-negative genus. In addition, the cap $B$ mutant can provide protection against substantial wild-type challenge that is equivalent to that of LVS. This work supports other reports on the feasibility of producing an effective genetically defined live $F$. tularensis vaccine from subspecies tularensis strain of $F$. tularensis, and provides a novel target that could be used in the generation of a multiple loci mutant for such a vaccine (Conlan et al., 2010).

\section{REFERENCES}

Alkhuder, K., Meibom, K. L., Dubail, I., Dupuis, M. \& Charbit, A. (2009). Glutathione provides a source of cysteine essential for intracellular multiplication of Francisella tularensis. PLoS Pathog 5, e1000284.

Anthony, L. S., Ghadirian, E., Nestel, F. P. \& Kongshavn, P. A. (1989). The requirement for gamma interferon in resistance of mice to experimental tularemia. Microb Pathog 7, 421-428.

Burke, D. S. (1977). Immunization against tularemia: analysis of the effectiveness of live Francisella tularensis vaccine in prevention of laboratory-acquired tularemia. J Infect Dis 135, 55-60.

Candela, T. \& Fouet, A. (2005). Bacillus anthracis CapD, belonging to the gamma-glutamyltranspeptidase family, is required for the covalent anchoring of capsule to peptidoglycan. Mol Microbiol 57, 717-726.

Candela, T. \& Fouet, A. (2006). Poly-gamma-glutamate in bacteria. Mol Microbiol 60, 1091-1098.

Candela, T., Moya, M., Haustant, M. \& Fouet, A. (2009). Fusobacterium nucleatum, the first Gram-negative bacterium demonstrated to produce polyglutamate. Can J Microbiol 55, 627-632. 
Chamberlain, R. E. (1965). Evaluation of live tularemia vaccine prepared in a chemically defined medium. Appl Microbiol 13, 232235.

Chen, W., Shen, H., Webb, A., KuoLee, R. \& Conlan, J. W. (2003). Tularemia in $\mathrm{BALB} / \mathrm{c}$ and $\mathrm{C} 57 \mathrm{BL} / 6$ mice vaccinated with Francisella tularensis LVS and challenged intradermally, or by aerosol with virulent isolates of the pathogen: protection varies depending on pathogen virulence, route of exposure, and host genetic background. Vaccine 21, 3690-3700.

Cherwonogrodzky, J. W., Knodel, M. H. \& Spence, M. R. (1994). Increased encapsulation and virulence of Francisella tularensis live vaccine strain (LVS) by subculturing on synthetic medium. Vaccine 12, 773-775.

Conlan, J. W., Sjostedt, A. \& North, R. J. (1994). CD4 ${ }^{+}$and $\mathrm{CD}^{+}{ }^{+}$Tcell-dependent and -independent host defense mechanisms can operate to control and resolve primary and secondary Francisella tularensis LVS infection in mice. Infect Immun 62, 5603-5607.

Conlan, J. W., Shen, H., Golovliov, I., Zingmark, C., Oyston, P. C., Chen, W., House, R. V. \& Sjostedt, A. (2010). Differential ability of novel attenuated targeted deletion mutants of Francisella tularensis subspecies tularensis strain SCHU S4 to protect mice against aerosol challenge with virulent bacteria: effects of host background and route of immunization. Vaccine 28, 1824-1831.

Drysdale, M., Heninger, S., Hutt, J., Chen, Y., Lyons, C. R. \& Koehler, T. M. (2005). Capsule synthesis by Bacillus anthracis is required for dissemination in murine inhalation anthrax. EMBO J 24, 221-227.

Eyles, J. E., Unal, B., Hartley, M. G., Newstead, S. L., Flick-Smith, H., Prior, J. L., Oyston, P. C. F., Randall, A., Mu, Y. \& other authors (2007). Immunodominant Francisella tularensis antigens identified using proteome microarray. Proteomics 7, 2172-2183.

Fortier, A. H., Slayter, M. V., Ziemba, R., Meltzer, M. S. \& Nacy, C. A. (1991). Live vaccine strain of Francisella tularensis: infection and immunity in mice. Infect Immun 59, 2922-2928.

Golovliov, I., Sjostedt, A., Mokrievich, A. \& Pavlov, V. (2003). A method for allelic replacement in Francisella tularensis. FEMS Microbiol Lett 222, 273-280.

Hood, A. M. (1977). Virulence factors of Francisella tularensis. J Hyg (Lond) 79, 47-60.

Khan, A. S., Morse, S. \& Lillibridge, S. (2000). Public-health preparedness for biological terrorism in the USA. Lancet 356, 11791182.

Kocianova, S., Vuong, C., Yao, Y., Voyich, J. M., Fischer, E. R., DeLeo, F. R. \& Otto, M. (2005). Key role of poly-gamma-DL-glutamic acid in immune evasion and virulence of Staphylococcus epidermidis. J Clin Invest 115, 688-694.

Larsson, P., Oyston, P. C. F., Chain, P., Chu, M. C., Duffield, M., Fuxelius, H.-H., Garcia, E., Hälltorp, G., Johansson, D. \& other authors (2005). The complete genome sequence of Francisella tularensis, the causative agent of tularemia. Nat Genet 37, 153-159.

Livak, K. J. \& Schmittgen, T. D. (2001). Analysis of relative gene expression data using real-time quantitative PCR and the $2^{-\Delta \Delta C}$ Method. Methods 25, 402-408.

Makino, S., Uchida, I., Terakado, N., Sasakawa, C. \& Yoshikawa, M. (1989). Molecular characterization and protein analysis of the cap region, which is essential for encapsulation in Bacillus anthracis. J Bacteriol 171, 722-730.

McCrumb, F. R. (1961). Aerosol infection of man with Pasteurella tularensis. Bacteriol Rev 25, 262-267.

Meibom, K. L., Forslund, A. L., Kuoppa, K., Alkhuder, K., Dubail, I., Dupuis, M., Forsberg, A. \& Charbit, A. (2009). Hfq, a novel pleiotropic regulator of virulence-associated genes in Francisella tularensis. Infect Immun 77, 1866-1880.
Merabishvili, M., Natidze, M., Rigvava, S., Brusetti, L., Raddadi, N., Borin, S., Chanishvili, N., Tediashvili, M., Sharp, R. \& other authors (2006). Diversity of Bacillus anthracis strains in Georgia and of vaccine strains from the former Soviet Union. Appl Environ Microbiol 72, 5631-5636.

Norqvist, A., Kuoppa, K. \& Sandström, G. (1996). Construction of a shuttle vector for use in Francisella tularensis. FEMS Immunol Med Microbiol 13, 257-260.

Olsufiev, N. G., Emelyanova, O. S. \& Dunayeva, T. N. (1959). Comparative study of strains of $B$. tularense in the old and new world and their taxonomy. J Hyg Epidemiol Microbiol Immunol 3, 138149.

Oyston, P. C. (2008). Francisella tularensis: unravelling the secrets of an intracellular pathogen. J Med Microbiol 57, 921-930.

Oyston, P. C. (2009). Francisella tularensis vaccines. Vaccine 27, D48D51.

Oyston, P. C. F., Sjöstedt, A. \& Titball, R. W. (2004). Tularaemia: bioterrorism defence renews interest in Francisella tularensis. Nat Rev Microbiol 2, 967-978.

Pechous, R. D., McCarthy, T. R., Mohapatra, N. P., Soni, S., Penoske, R. M., Salzman, N. H., Frank, D. W., Gunn, J. S. \& Zahrt, T. C. (2008). A Francisella tularensis Schu S4 purine auxotroph is highly attenuated in mice but offers limited protection against homologous intranasal challenge. PLOS ONE 3, e2487.

Qin, A., Scott, D. W. \& Mann, B. J. (2008). Francisella tularensis subsp. tularensis Schu S4 disulfide bond formation protein B, but not an RND-type efflux pump, is required for virulence. Infect Immun 76, 3086-3092.

Qin, A., Scott, D. W., Thompson, J. A. \& Mann, B. J. (2009). Identification of an essential Francisella tularensis subsp. tularensis virulence factor. Infect Immun 77, 152-161.

Ravel, J., Jiang, L., Stanley, S. T., Wilson, M. R., Decker, R. S., Read, T. D., Worsham, P., Keim, P. S., Salzberg, S. L. \& other authors (2009). The complete genome sequence of Bacillus anthracis Ames "Ancestor". J Bacteriol 191, 445-446.

Sambrook, J., Fritsch, E. F. \& Maniatis, T. (1989). Molecular Cloning: a Laboratory Manual. Cold Spring Harbor, NY: Cold Spring Harbor Laboratory.

Sandström, G., Löfgren, S. \& Tärnvik, A. (1988). A capsule-deficient mutant of Francisella tularensis LVS exhibits enhanced sensitivity to killing by serum but diminished sensitivity to killing by polymorphonuclear leukocytes. Infect Immun 56, 1194-1202.

Saslaw, S., Eigelsbach, H. T., Prior, J. A., Wilson, H. E. \& Carhart, S. (1961a). Tularemia vaccine study. II. Respiratory challenge. Arch Intern Med 107, 702-714.

Saslaw, S., Eigelsbach, H. T., Wilson, H. E., Prior, J. A. \& Carhart, S. (1961b). Tularemia vaccine study. I. Intracutaneous challenge. Arch Intern Med 107, 689-701.

Simon, P., Priefer, U. \& Pühler, A. (1983). A broad host range mobilization system for in vivo genetic engineering: transposon mutagenesis in Gram negative bacteria. Nat Biotechnol 1, 784-791.

Straskova, A., Pavkova, I., Link, M., Forslund, A.-L., Kuoppa, K., Noppa, L., Kroca, M., Fucikova, A., Klimentova, J. \& other authors (2009). Proteome analysis of an attenuated Francisella tularensis $d s b A$ mutant: identification of potential DsbA substrate proteins. J Proteome Res 8, 5336-5346.

Su, J., Yang, J., Zhao, D., Kawula, T. H., Banas, J. A. \& Zhang, J. R. (2007). Genome-wide identification of Francisella tularensis virulence determinants. Infect Immun 75, 3089-3101.

Tärnvik, A. (1989). Nature of protective immunity to Francisella tularensis. Rev Infect Dis 11, 440-451. 
Thomas, R. M., Titball, R. W., Oyston, P. C. F., Griffin, K., Waters, E., Hitchen, P. G., Michell, S. L., Grice, I. D., Wilson, J. C. \& Prior, J. L. (2007).

The immunologically distinct $\mathrm{O}$ antigens from Francisella tularensis subspecies tularensis and Francisella novicida are both virulence determinants and protective antigens. Infect Immun 75, 371-378.

Twine, S., Byström, M., Chen, W., Forsman, M., Golovliov, I., Johansson, A., Kelly, J., Lindgren, H., Svensson, K. \& other authors
(2005). A mutant of Francisella tularensis strain SCHU S4 lacking the ability to express a 58-kilodalton protein is attenuated for virulence and is an effective live vaccine. Infect Immun 73, 8345-8352.

Weiss, D. S., Brotcke, A., Henry, T., Margolis, J. J., Chan, K. \& Monack, D. M. (2007). In vivo negative selection screen identifies genes required for Francisella virulence. Proc Natl Acad Sci U S A 104, 6037-6042. 